\title{
Fenología reproductiva de Stenocereus thurberi (Cactaceae) en una región de transición del norte de Sinaloa, México
}

\section{Reproductive phenology of Stenocereus thurberi (Cactaceae) in a transitional region of northern Sinaloa, Mexico}

\author{
Bladimir Salomón-Montijo, Álvaro Reyes-Olivas* \& Bardo H. Sánchez-Soto \\ Colegio de Ciencias Agropecuarias, Facultad de Agronomía del Valle del Fuerte, Universidad Autónoma de Sinaloa, Calle 16 \\ y Avenida Japaraqui, C.P. 81110, Juan José Ríos, Ahome, Sinaloa, México. \\ *alreo.uas@gmail.com
}

\begin{abstract}
RESUMEN
Los patrones fenológicos, moldeados por las interacciones físicas y bióticas en hábitats marginales, pueden revelar características únicas para la permanencia de una población. La fenología de Stenocereus thurberi, una cactácea columnar del Desierto Sonorense, ha mostrado variación geográfica asociada con el clima y los polinizadores, pero no se conoce su comportamiento en ambientes más húmedos de su área de distribución. El objetivo de esta investigación es describir la dinámica reproductiva y la estructura de tamaños de tres poblaciones en una transición matorral-bosque caducifolio de $170 \mathrm{~km}$ de longitud. Las plantas se caracterizaron por su talla, cobertura y número de tallos; los estados fenológicos se basaron en la escala de Campbell y se registraron quincenalmente en 50 individuos de cada población. Se encontraron diferencias en la arquitectura de las plantas, la distribución de tamaños y la actividad reproductiva. El dosel individual en plantas de la población costera (Plan de Guadalupe, $244 \mathrm{~mm}$ ) es más ramificado $(23.3 \pm 3.0$ tallos / planta) y más extendido ( $4.6 \pm 0.7 \mathrm{~m}^{2}$; media \pm 1 error estándar) que en el bosque caducifolio (Las Cruces, $830 \mathrm{~mm}$ ), cuyas plantas tuvieron mayor desarrollo vertical $\left(9.3 \pm 1.3\right.$ tallos / planta, $\left.1.2 \pm 0.2 \mathrm{~m}^{2}\right)$. Las poblaciones mostraron diferencias en la estructura de tamaños, con plantas más grandes en la costa que en las otras poblaciones. El calendario del periodo reproductivo se demoró 15-30 días y se prolongó por cuatro semanas más en la costa que en las otras poblaciones. La producción de estructuras reproductivas en fechas pico mostró diferencias altamente significativas $(\mathrm{F}=31.68, p<$ 0.001), con medias de $69.5 \pm 8.7$ en la costa, $28.3 \pm 3.2$ en Buenavista y $16.6 \pm 5.4$ en Las Cruces. La precipitación y la temperatura son determinantes en la producción de estructuras reproductivas y la estructura de tamaños de las poblaciones locales. Es posible que la baja producción de flores y frutos de pitaya en el bosque caducifolio sea consecuencia de altas tasas de aborto, depredación de estructuras y competencia por luz, mientras la polinización es facilitada por coexistencia con otra especie columnar, Stenocereus montanus.
\end{abstract}

Palabras clave: Reproducción, fenología, Stenocereus thurberi, llanura costera, pie de montaña.

\begin{abstract}
Phenological patterns, which are molded by physical and biotic interactions, in marginal habitats could reveal peculiar characteristics for the permanence of a population. Phenology of Stenocereus thurberi, a columnar cactus from the Sonoran Desert, has shown geographic variation that is associated with the weather and pollinators, however, there is no information regarding its behavior in habitats that are more humid than its core distribution area. The aim of this study was to describe the reproductive dynamics and the size structure of three populations along a scrub-tropical dry forest transition $170 \mathrm{~km}$ in length. Plants were characterized by height, coverage and number of stems. Phenological stages were based on the Campbell scale and were registered fortnightly on 50 individuals from each population. We found differences in the individual architecture, plant size distribution and reproductive activity. Canopy structure in plants from the coastal population (Plan de Guadalupe, $244 \mathrm{~mm})$ is more branched $\left(23.3 \pm 3.0\right.$ stems/plant) and wider $\left(4.6 \pm 0.7 \mathrm{~m}^{2}\right)$ than the population from the deciduous forest (Las Cruces, $830 \mathrm{~mm}$ ), on which plants showed higher vertical development $(9.3 \pm 1.3$ stems / plant, $1.2 \pm 0.2 \mathrm{~m}^{2}$ ). Populations exhibited differences in the size structure, with bigger plants on the coast than the other populations. Timing of the breeding season was delayed 15-30 days and was extended four more weeks in the coast than in the other populations. Production of reproductive structures in peak dates showed highly significant differences (F $=31.68, p<0.001$ ), with a mean of $69.5 \pm 8.7$ in the coast, $28.3 \pm 3.2$ in Buenavista and $16.6 \pm 5.4$ in Las Cruces. Rainfall and temperature are key determinants in the production of reproductive structures and in the size structure of the local
\end{abstract}


populations. It is likely that the low production of pitaya flowers and fruits in the deciduous forest be a consequence of high abortion rates, predation of structures and light competition, while pollination is improved by facilitation from other columnar cactus, Stenocereus montanus.

KEYwORDs: Reproduction, phenology, Stenocereus thurberi, coastal plain, piedmont.

\section{INTRODUCCIÓN}

Los patrones de floración y fructificación de las plantas están moldeados por la actividad de los polinizadores, los depredadores de flores y frutos, los dispersores de las semillas y los competidores. Sin embargo, en medios secos es más probable que la variación de estos patrones se atribuya a factores físicos, principalmente la temperatura y la disponibilidad de agua (Forrest \& Miller-Rushing 2010; Wang et al. 2016). Estos factores influyen a escala de hábitats y poblaciones y también a nivel de los parches o micrositios donde se distribuyen los individuos de la población. Además, en hábitats marginales o periféricos del área de distribución, las poblaciones tienden a ser más pequeñas, aisladas y más divergentes que las poblaciones centrales; las presiones selectivas del clima, el suelo, y la comunidad suelen ser distintos, por lo que pueden revelar características únicas que no son observables en hábitats favorables (Soule 1973; Leppig \& White 2006; Kawecki 2008).

Las poblaciones de Stenocereus thurberi (Engelm.) Buxb. ("pitaya dulce"), una cactácea del Desierto Sonorense apreciada por sus frutos y madera (Yetman \& Van Devender 2002), se extienden ampliamente en los matorrales costeros y marginalmente en los bosques tropicales secos de Sinaloa. Esta vegetación se ha visto fuertemente afectada por el cambio de uso de suelo, causado por el crecimiento de las actividades agropecuarias, con una pérdida de recurso forestal de 11,379 ha año-1 (Céspedes- Flores \& MorenoSánchez 2010). Sin embargo no hay información sobre las poblaciones locales de pitaya en estas zonas de transición que bordean el Desierto Sonorense, aunque hay estudios sobre sus interacciones con nodrizas (Arriaga et al. 1993, Reyes-Olivas et al. 2002), germinación (Sánchez-Soto et al. 2005, Sánchez-Soto et al. 2010) e incidencia de viviparidad (Cota-Sánchez et al. 2007, Pérez-González et al. 2015).

La limitación de polinizadores, uno de los factores causantes de la variación anual en la producción de frutos, está bien documentada en poblaciones de pitaya del Desierto Sonorense (Fleming et al. 1996, Fleming 2000, Sahley 2001, Bustamante et al. 2010). Otra parte de la variación está relacionada con la estructura de tamaños de la población y los regímenes de lluvia y temperatura, que son determinantes en los tiempos de inicio de la floración, la duración del periodo reproductivo y la producción de estructuras reproductivas (Parker 1987, 1993, Bustamante \& Búrquez 2008). Además, la producción de frutos se ha visto afectada por la disponibilidad de polen heteroespecífico, lo que constituye un fenómeno raro en $S$. thuberi, porque generalmente el polen extraño conduce al aborto de flores (Fleming 2000, 2006). ¿Qué semejanzas fenológicas comparten las poblaciones de Sinaloa con las del Desierto Sonorense, y cómo estos factores pueden afectar la dinámica de reproducción de la cactácea?, sobre todo ¿cómo difieren los patrones fenológicos en el bosque tropical caducifolio, identificado como el extremo más húmedo de su distribución ecológica?

El objetivo de la presente investigación es comparar la fenología reproductiva de Stenocereus thurberi en tres ambientes contrastantes de la transición del matorral al bosque caducifolio del norte de Sinaloa. Nuestros datos están limitados a un ciclo reproductivo y no son suficientes para hacer un análisis numérico de las correlaciones ecológicas, por lo tanto nos limitaremos a formular algunas hipótesis sobre los patrones observados. Esperamos encontrar amplias diferencias morfológicas en la arquitectura de las plantas y un comportamiento fenológico más asincrónico en la población costera por ser el ambiente con menor oscilación térmica. El periodo reproductivo podría iniciar primero y tener mayor duración en esta población, en concordancia con los patrones que han observado otros autores (Bustamante \& Búrquez 2008, Figueiredo-Goulart 2005). En el bosque caducifolio cabría esperar un adelanto en el periodo reproductivo acorde con el patrón de las temperaturas y una mayor restricción en la producción de estructuras reproductivas.

\section{MATERIALES Y MÉTODOS}

\section{ESPECIE DE ESTUDIO}

Stenocereus thurberi (Cactaceae) tiene tallos columnares de 3 a $8 \mathrm{~m}$ de altura, ramificados desde la base o con un tronco corto. Sus flores, de 6 a $8 \mathrm{~cm}$ de largo son nocturnas, hermafroditas, tubulares, polinizadas regularmente por murciélagos; éstas abren al atardecer y cierran a la mañana siguiente, por lo que pueden recibir a otros polinizadores como aves e insectos. El fruto es una baya de 4 a $7.5 \mathrm{~cm}$ de largo, con pulpa rojiza y semillas de color negro brillante (Turner et al. 1995), alrededor de 2000 semillas por fruto (Parker 1987); su reproducción es sexual.

La pitaya se distribuye desde el norte de Sinaloa y oeste de Chihuahua hasta el sudoeste de Arizona y la mitad sur de la península de Baja California. En el extremo norte de la 
distribución (Arizona) los individuos tienen frecuentemente constricciones y fisuras en los tallos causados por heladas, principal factor que limita su distribución septentrional (Parker 1988, Turner et al. 1995). Por lo mismo, las plantas están restringidas a las pendientes rocosas y bordes de acantilados, donde la radiación nocturna de calor las protege de las heladas (Parker 1988). En las llanuras costeras del Desierto Sonorense y Sinaloa algunos individuos alcanzan alturas entre $12 \mathrm{y}$ hasta $15 \mathrm{~m}$, mientras que las poblaciones septentrionales tienen tallas menores (Turner et al. 1995).

\section{DESCRIPCIÓN DEL ÁREA DE ESTUDIO}

El estudio se realizó en tres poblaciones naturales del norte de Sinaloa, localizadas sobre un gradiente climático de $170 \mathrm{~km}$ de longitud, desde la costa cercana al puerto de Topolobampo hasta el piedemonte de la Sierra Madre Occidental (Fig. 1). La primera población (PG) se localiza en dunas costeras del Ejido Plan de Guadalupe (25\%41'32,77" N y $109^{\circ} 08^{\prime} 54,59^{\prime \prime}$ O, 0 msnm), a $12 \mathrm{~km}$ del puerto citado, con una precipitación media anual de 244.1 $\mathrm{mm}$, temperatura media anual de $24,4^{\circ} \mathrm{C}$, media máxima de $30,6^{\circ} \mathrm{C}$ y media mínima de $20,8^{\circ} \mathrm{C}$. Según la clasificación de suelos FAO-UNESCO (INEGI 1988), el tipo de suelo es Solonchak y su vegetación dominante es matorral xerófilo (Rzedowski 1978); entre las especies comunes se encuentran: Fouquieria macdougallii Nash, Jatropha cinerea (Ortega) Müll.Arg., Prosopis yaquiana R.A.Palacios. La segunda población (BV) se localiza en Buenavista, Municipio de El Fuerte (26 $04^{\prime}$ '00,45" N y $\left.108^{\circ} 46^{\prime} 53,96^{\prime \prime} \mathrm{O}, 150 \mathrm{msnm}\right)$, en lomeríos del valle agrícola, $6 \mathrm{~km}$ al norte de San Blas. La estación meteorológica "Bocatoma" localizada a $5 \mathrm{~km}$ del sitio de estudio registra una media anual de precipitación de $472.3 \mathrm{~mm}$, una temperatura media anual de $23,8{ }^{\circ} \mathrm{C}$, media máxima de $33,1^{\circ} \mathrm{C}$ y media mínima de $17,6{ }^{\circ} \mathrm{C}$. El suelo es de tipo Litosol y la vegetación es un bosque espinoso con diversas especies leguminosas: Guaiacum coulteri A.Gray, Caesalpinia platyloba S.Watson y Acacia farnesiana (L.) Willd, entre otras. La tercera población (LC) se encuentra en Las Cruces (26 $56^{\circ}$ ' 18,60" N y $108^{\circ} 23^{\prime} 12,19^{\prime}$ ” O, 350 msnm), $18 \mathrm{~km}$ al norte de la cabecera municipal de Choix. Corresponde al pie de montaña, la parte más alta y húmeda del gradiente. La estación meteorológica más cercana, "Huites", registra una precipitación media anual de $830 \mathrm{~mm}$ y temperatura media anual de $27,31^{\circ} \mathrm{C}$, media máxima de $35,1{ }^{\circ} \mathrm{C}$ y una media mínima de $16,1{ }^{\circ} \mathrm{C}$. El tipo de suelo característico es el Litosol y la vegetación dominante es el bosque tropical caducifolio, siendo especies comunes Caesalpinia eriostachys Benth, Lysiloma acapulcense (Kunth) Benth y Ceiba pentandra (L.) Gaertn (Fig. 1).

\section{ESTRUCTURA DE TAMAÑOS Y FENOLOGÍA}

El estudio se realizó en el periodo reproductivo de abril a octubre del 2014. En cada localidad se seleccionaron al azar 50 individuos reproductivos y aparentemente sanos, los cuales fueron georreferenciados y marcados con etiquetas de aluminio para facilitar su relocalización. Los muestreos fueron quincenalmente, desde el inicio de floración hasta el fin de la fructificación. Se registraron tres estadios de

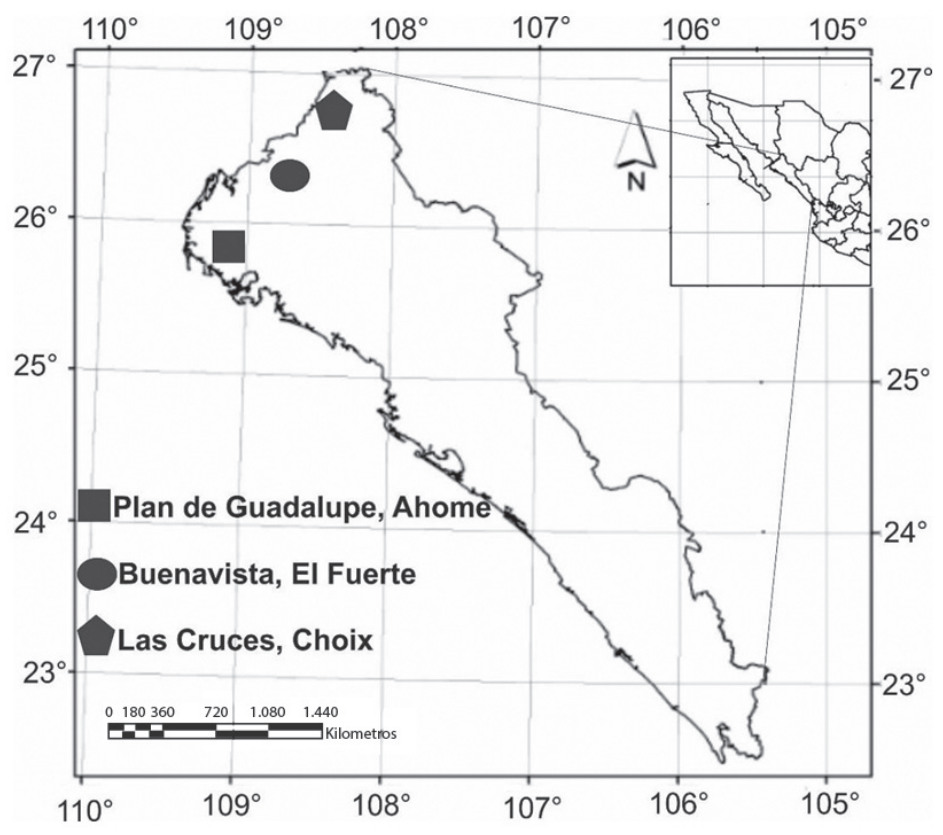

FIgURA 1. Localización de las tres poblaciones de muestreo.

FIGURE 1. Location of the three sample populations. 
acuerdo con la escala de Campbell (Rondón 1994): botones, flores y frutos. Los botones se consideraron desde que fueron visibles hasta preantesis; en el estado de flor se incluyeron las que estaban parcial o totalmente abiertas; los frutos se contaron desde el estado incipiente (flores que permanecieron deshidratadas en la planta después de la polinización) hasta la dehiscencia. Para caracterizar la población por tamaños se registró la altura, la cobertura del dosel y el número de tallos. La variable más correlacionada con la producción de estructuras reproductivas se usó para categorizar tamaños. La altura se midió con un clinómetro, desde la base hasta la punta del tallo más alto; la cobertura del dosel se determinó por los ejes de la elipse formada por la proyección vertical del dosel a la superficie del suelo (Mueller-Dombois \& Ellenberg 1974). En el recuento de los tallos se incluyeron aquellos que tenían más de $0,3 \mathrm{~m}$ de longitud, cuando usualmente son reproductivos.

\section{AnÁlisis De Datos}

Con base en la metodología de Figueiredo-Goulart (2005), las plantas se clasificaron por su estado fenológico, definido por la combinación de frecuencias relativas de sus estructuras reproductivas (Fournier 1974): 0, ausencia de estructuras; 2 , $1-25 \%$; 3, 26-50 \%; 4, 76-100 \%. El índice de diversidad de Shannon-Wiener, usual en ecología de comunidades, se adaptó usando los estados fenológicos como "especies" para comparar la sincronía reproductiva de las poblaciones. Esta misma lógica se usó para estimar los índices de semejanza de Morisita-Horn entre ellas.

La relación de la producción de estructuras reproductivas y el tamaño de las plantas se determinó por correlaciones de Pearson y análisis de varianza. La distribución de tamaños de las poblaciones se comparó mediante pruebas de independencia con la prueba de probabilidad exacta de
Fisher (Siegel 1985). Generalmente los datos fenológicos no cumplen con los requerimientos del ANOVA de medidas repetidas por presentar una estructura desbalanceada y carecer de normalidad. Para remediar el problema se redujo el análisis a siete de las trece fechas registradas en el periodo álgido de reproducción; el número de estructuras reproductivas se transformó con $\log 10(\mathrm{x}+1)$ y logró normalidad en los datos con la prueba de Anderson-Darling (Tamhane 2009). Los efectos intrasujetos se juzgaron con F ajustadas por el épsilon de Greenhouse-Geisser, una opción cuando falta esfericidad en la matriz de covarianzas (BlancaMena, 2004). Estos análisis se hicieron con el software SAS (SAS Institute 2011).

\section{RESULTADOS}

Producción De ESTRUCTURAS REPRODUCTIVAS

Se registraron densidades de 59, 138 y 56 plantas/ha en las poblaciones $\mathrm{PG}, \mathrm{BV}$ y LC, respectivamente; en el mismo orden, los individuos reproductivos representaron el 79, 92,7 y $70,7 \%$ de la población. Las tres poblaciones tienen bajas tasas de mortalidad de tallos, ligeramente mayores en LC que en PG y BV ( $3,1 \%$ vs $1,3 \%$ y $0,52 \%)$.

Las tallas de las plantas variaron de 2,10 a $7,90 \mathrm{~m}$, con promedio de $4,1 \mathrm{~m}$. La población LC, situada a mayor altitud y con mayor precipitación promedio que los otros dos sitios, presentó una media de $4,5 \mathrm{~m}$. La población costera (PG) tuvo tallas menores y la de valle (BV) valores intermedios (Tabla I). La cobertura individual varió de 0,02 a 23,33 $\mathrm{m}^{2}$ y promedió $2,5 \mathrm{~m}^{2}$; fue mayor en la población PG $\left(4,6 \mathrm{~m}^{2}\right)$, menor en la LC e intermedia en la BV. El número de tallos por individuo varió de 1 a 105 y promedió 15,1; en la población PG promedió 23,3 y en la LC menos

Tabla I. Estadísticos descriptivos de tres variables de tamaños de S. thurberi $(\mathrm{n}=50)$ en poblaciones del norte de Sinaloa, México.

TABLE I. Descriptive statistics of three size variables for S. thurberi $(\mathrm{n}=50)$ in populations of northern Sinaloa, México.

\begin{tabular}{ccrrr}
\hline \multirow{2}{*}{ Localidad } & Tamaños & Media \pm e. e. & Mínima & Máxima \\
& & & & \\
\hline \multirow{3}{*}{ Plan de Guadalupe } & Talla $(\mathrm{m})$ & $3,9 \pm 0,1$ & 2,14 & 6,40 \\
& Cobertura $\left(\mathrm{m}^{2}\right)$ & $4,6 \pm 0,7$ & 0,07 & 23,33 \\
& Número de tallos & $23,3 \pm 3,0$ & 1,00 & 105,00 \\
& Talla $(\mathrm{m})$ & $4,2 \pm 0,1$ & 2,30 & 6,50 \\
Buenavista & Cobertura $\left(\mathrm{m}^{2}\right)$ & $1,8 \pm 0,3$ & 0,12 & 11,04 \\
& Número de tallos & $12,8 \pm 1,3$ & 2,00 & 56,00 \\
& Talla $(\mathrm{m})$ & $4,6 \pm 0,2$ & 2,10 & 7,90 \\
Las Cruces & Cobertura $\left(\mathrm{m}^{2}\right)$ & $1,2 \pm 0,2$ & 0,02 & 9,62 \\
& Número de tallos & $9,3 \pm 1,3$ & 1,00 & 54,00 \\
\hline
\end{tabular}


Fenología y estructura de tamaños de Stenocereus thurberi: SAlomon-MontiJo, B. ET AL.

de diez. Las correlaciones de Pearson indican asociación de la producción de estructuras reproductivas (botones, flores y frutos) con las tres variables morfométricas. Sin embargo, la correlación es mayor con el número de tallos y con la cobertura del dosel en las tres poblaciones. En la población de LC, la correlación con el número de tallos fue $r=0,813$ para botones, $r=0,693$ para flores y $r=0,821$ para frutos, semejantes a los de la cobertura; en la población PG las correlaciones fueron de 0,696, 0,661 y 0,751, respectivamente.

Las categorías de tamaño de las poblaciones se escalaron a la distribución lognormal por conveniencia estadística para evitar ceros en los cuadros de contingencia. Las categorías fueron: (1) 1-5, (2) 6-11, (3) 12-23, (4) 24-48, (5) 49+. Las plantas de las categorías 1 y 2 representan el $50 \%$ de la población en Las Cruces y $24 \%$ en las otras poblaciones, mientras que las categorías 4 y 5 representaron el $54 \%$ de la población de Plan de Guadalupe y 12-14 \% en Buenavista y Las Cruces (Fig. 2). Con esta información se infiere que las poblaciones difieren en su estructura de tamaños $(\mathrm{P}<$ 0,0001, Prueba Exacta de Fisher).

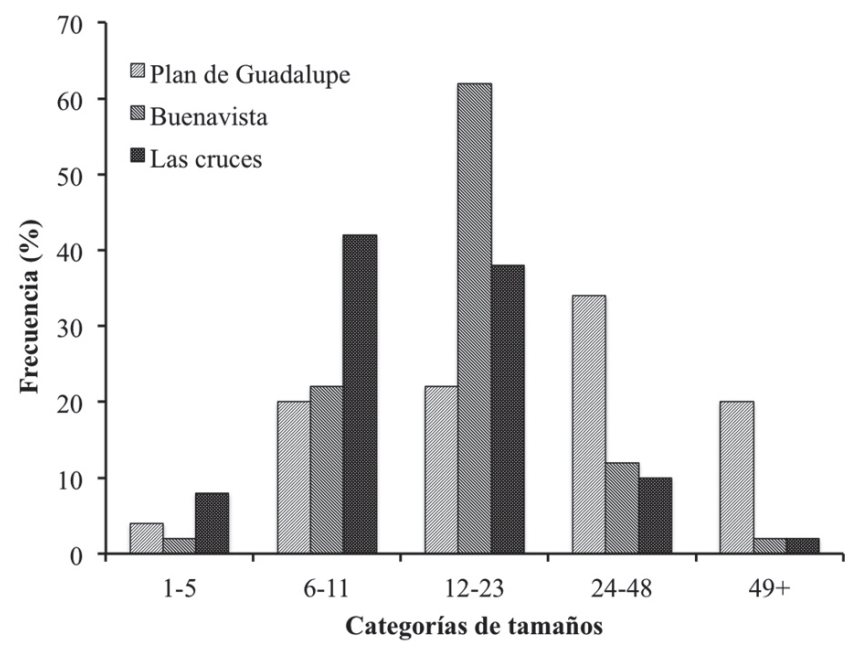

Figura 2. Frecuencia del número de tallos en cinco categorías de tamaños, en tres poblaciones del norte de Sinaloa.

FigurE 2. Frequency of stems in five size categories, in three populations in northern Sinaloa.

TABLA II. Análisis de varianza de medidas repetidas para probar el efecto de poblaciones, tamaño de plantas y tiempo de muestreo en la producción de estructuras reproductivas (botones, flores y frutos) de la pitaya dulce Stenocereus thurberi en el norte de Sinaloa.

TABLE II. Repeated measures analysis of variance to test the effect of population, size of plants and sampling time in the production of reproductive structures (buttons, flowers and fruits) of organ pipe cactus Stenocereus thurberi in northern Sinaloa.

\begin{tabular}{|c|c|c|c|c|}
\hline FUENTE DE VARIACIÓN & G. L. & MS & $\mathrm{F}$ & $\mathrm{P}>\mathrm{F}$ \\
\hline \multicolumn{5}{|l|}{ ENTRE SUJETOS } \\
\hline Población & 2 & 7,164 & 12,32 & $<0,0001$ \\
\hline Tamaños & 4 & 16,028 & 27,57 & $<0,0001$ \\
\hline Población *tamaños & 8 & 0,466 & 0,80 & 0,6023 \\
\hline Error & 135 & 0,581 & & \\
\hline INTRA-SUJETOS & & & & Adj $\mathrm{P}>\mathrm{F}$ \\
\hline Tiempo & 6 & 5,075 & 60,51 & $<0,0001$ \\
\hline Tiempo*Población & 12 & 1,148 & 13,68 & $<0,0001$ \\
\hline Tiempo*Tamaños & 24 & 0,268 & 3,20 & 0,0004 \\
\hline Tiempo*Pob*Tam & 48 & 0,162 & 1,93 & 0,0076 \\
\hline Error & 810 & 0,084 & & \\
\hline
\end{tabular}

La producción de estructuras reproductivas tiene diferencias altamente significativas entre poblaciones $(\mathrm{F}=12,32, \mathrm{P}<0,0001)$ y entre categorías de tamaños ( $F=27,57, P<0,0001)$, pero no hubo interacción entre ambos factores $(\mathrm{F}=0,8, \mathrm{P}=0,602)$. Los efectos intra-sujetos son altamente significativos para el factor repetido (tiempo) y las interacciones tiempo*población, tiempo*categorías y tiempo*población*categorías $(\mathrm{P}<0,0001, \mathrm{~F}$ ajustada $)$ (Tabla II). En fechas pico las poblaciones alcanzaron una producción de $69,5 \pm 8,7$ estructuras reproductivas en la población PG, 28,3 $\pm 3,2$ en BV y 16,6 $\pm 5,4$ en LC.

\section{FENOLOGÍA}

El periodo reproductivo duró seis meses (abril-septiembre) en las poblaciones $\mathrm{BV}$ y LC, y siete meses en la población PG. El registro de botones florales inició en abril en las tres poblaciones, terminó en agosto en la BV y LC, y se extendió hasta septiembre en la PG. El punto más álgido de esta fase se alcanzó en mayo y junio, con mayor producción en la población PG (Fig. 3). La apertura de flores tuvo un comportamiento similar al de los botones, con picos máximos en mayo y junio, siendo mayor en la población costera (PG) y menor en la población de piedemonte (LC) 
(Fig. 4). Los puntos álgidos de la producción de frutos se dieron en junio en las poblaciones BV y LC, y en julio en la PG, los meses más calientes del periodo de estiaje (Fig. 5). La producción de estructuras reproductivas fue unimodal y asincrónica en las tres poblaciones.

La diversidad (asincronía) fenológica reproductiva de las poblaciones fue de $1,87 \pm 0,9$ en $\mathrm{PG}, 1,73 \pm 1,2$ en $\mathrm{BV} \mathrm{y}$ 1,2 $\pm 1,0$ en LC. El índice de Morisita-Horn reveló un alto grado de semejanza de las poblaciones, ligeramente mayor entre BV-LC $(\mathrm{MH}=0,87)$ y BV-PG $(\mathrm{MH}=0,82)$ que entre las poblaciones $\mathrm{PG}-\mathrm{LC}(\mathrm{MH}=0,72)$.

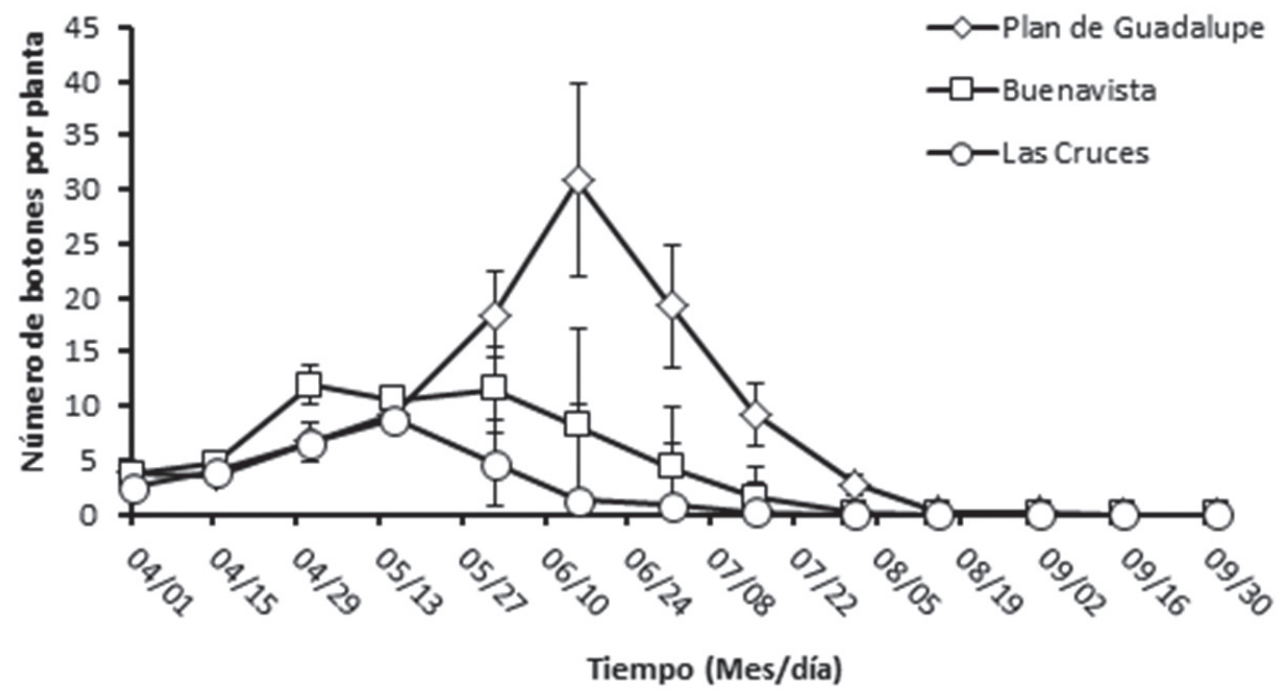

FIGURA 3. Dinámica de la producción de botones por planta de $S$. thurberi, en tres poblaciones del norte de Sinaloa.

Figure 3. Production dynamics of buttons per plant of $S$. thurberi, in three populations in northern Sinaloa.

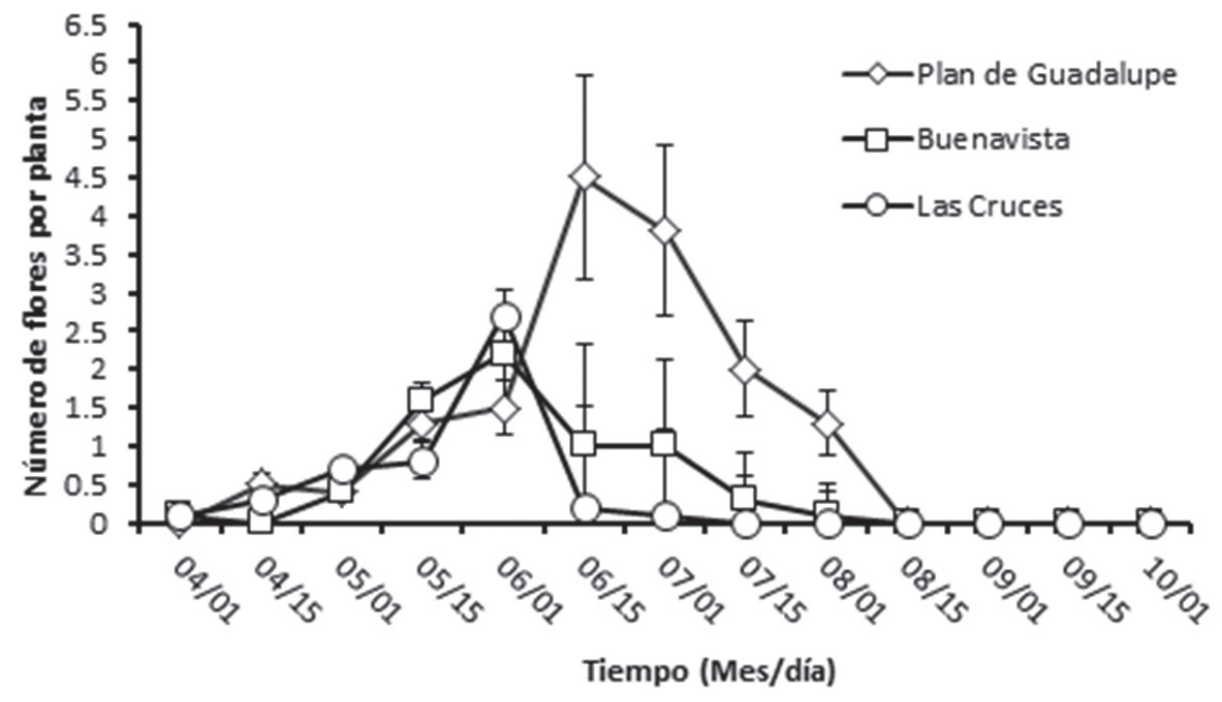

FIgURA 4. Variación en la producción de flores por planta de S. thurberi, en tres poblaciones del norte de Sinaloa, México.

FIGURE 4. Flower production variation per plant of S. thurberi, in three populations in northern Sinaloa, México. 


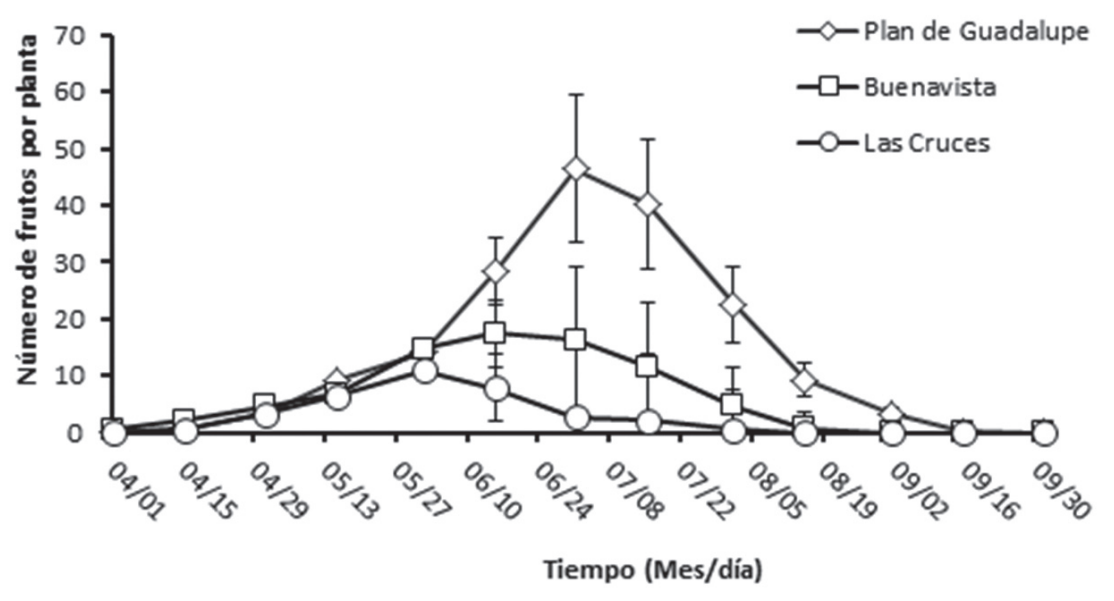

FIGURA 5. Variación en la producción de frutos por planta de S. thurberi, en tres poblaciones del norte de Sinaloa, México.

FIGURE 5. Fruit production variation per plant of S. thurberi, in three populations of northern Sinaloa, México.

\section{DISCUSIÓN}

La arquitectura alargada y poco ramificada de $S$. thurberi en Las Cruces es acorde con lo observado en otros cactos columnares que crecen en bosque tropical caducifolio; las plantas son significativamente más altas que en poblaciones de Arizona, el límite septentrional de distribución, donde el 92\% de los individuos tienen menos de 2,5 m de alto (Parker 1987). En contraste, las plantas de la población costera de Plan de Guadalupe tienen mayor expansión lateral y más tallos, como es usual en poblaciones costeras de Sonora (Bustamante 2003, Yetman 2006). Tales diferencias son función de las condiciones climáticas y edáficas, en respuesta a la estatura y densidad de la vegetación asociada (Cody 1986, Cornejo \& Simpson 1997).

Los elementos de la población contribuyen diferente en la reproducción de acuerdo a su edad o tamaño, este es un conocimiento que se ha incorporado en los estudios demográficos para determinar tasas de cambio, estimar el valor reproductivo de los individuos y proyectar el futuro de la población (Gotelli 2008). Este enfoque está en auge en las cactáceas, entre ellas Carnegiea gigantea (Engelm.) Britton \& Rose, Pachycereus schottii (Engelm.) D.R. Hunt, Neobuxbaumia tetetzo (F.A.C. Weber ex K. Schum.) Backeb., N. macrocephala (F.A.C. Weber ex K. Schum.) E.Y. Dawson, Echinocactus platyacanthus Link \& Otto y Harrisia portoricensis Britton y Harrisia portoricensis (Steenbergh \& Lowe 1977, Parker 1989, Godínez-Álvarez et al. 1999, Esparza-Olguín et al. 2002, Díaz-Hernández et al. 2008, Rojas-Sandoval \& Meléndez-Ackerman 2013). En Carnegiea gigantea, Stenocereus griseus (Haw.) Buxb., Subpilocereus repandus (L.) Backeb. y Pilosocereus lanuginosus (L.) Byles \& G.D. Rowley la fecundidad está asociada al número de tallos (Steenbergh \& Lowe 1977, Petit 2001). Esto es debido a que las plantas más grandes tienen mayor área fotosintética y tejido suculento para almacenar agua que las plantas pequeñas, por lo tanto son capaces de acumular y asignar más recursos a la función reproductiva (Nobel 2002).

La producción de estructuras reproductivas tiene un valor indicativo de la fecundidad. Las plantas de la población costera (PG) fueron 1,8 veces más grandes por su número de tallos reproductivos que la población de valle (BV) y 2,5 veces más que la población de piedemonte (LC); la población PG tuvo mayor proporción (54\%) de plantas con más de 24 tallos, mientras que las poblaciones BV y LC tuvieron 12-14 $\%$ de las plantas con estas dimensiones. Consecuentemente, en las fechas pico, la población costera produjo 2,5 veces más estructuras reproductivas que la población del valle y 4,2 veces más que la población de piedemonte. Al parecer, las tres poblaciones tienen capacidad para crecer debido a que muestran una distribución lognormal de tamaños (sesgada a la derecha), una propiedad común en las poblaciones resultante de la desigualdad biológica entre individuos (Damgaard \& Weiner 2000). Este tipo de distribución regularmente está asociado con poblaciones en crecimiento que tienen tasas de incorporación constantes (Medel-Narváez et al. 2006).

La población de Las Cruces tiene aproximadamente la mitad de los tallos que la población costera y produce un cuarto de las estructuras reproductivas de esa población. Esta desproporción sugiere otros factores implicados además del tamaño. Uno de ellos podría ser la competencia por recursos (luz), ya que hay indicios de plantas más ramificadas en espacios abiertos del bosque caducifolio. Se ha observado que $S$. thurberi crece asociada con arbustos nodriza en 
bosque caducifolio (Arriaga et al. 1993) o asociada con piedras en matorrales abiertos (Reyes-Olivas et al. 2002), pero no existe evidencia de que pueda independizarse y es improbable que suceda en este ambiente. Otro factor posible del que se tienen indicios en campo es la depredación y aborto de estructuras por aves e insectos. Algunos autores han documentado competencia por polinizadores en el Desierto Sonorense (Sahley 2001, Fleming et al. 1996), sin embargo la baja densidad y aislamiento relativo de la población de Las Cruces podría experimentar interacción facilitativa de otra cactácea columnar, Stenocereus montanus (Britton \& Rose) Buxb., como posible atrayente de polinizadores.

El periodo reproductivo en la población costera de Sinaloa es un mes más amplio que en el sur de Sonora, posiblemente por el incremento de las temperaturas medias máximas de otoño-invierno (Bustamante \& Búrquez 2008). El inicio de floración de las especies de Stenocereus se desplaza de sur a norte: S. queretaroensis (F.A.C. Weber) Buxb. produce estructuras reproductivas de enero a julio en el centro de México (Gudiño \& De la Barrera 2014, Rodríguez-Oseguera et al. 2012), unos meses antes que $S$. thurberi en el noroeste del país. Este proceso, regulado por las temperaturas, está acoplado con los tiempos de migración de los murciélagos polinizadores (Fleming et al. 2001).

El comportamiento unimodal de la curva fenológica, con máxima floración en la época seca, es común en cactáceas columnares (Fleming et al. 2001, Pavón \& Briones 2001, Bustamante \& Búrquez 2008). En esta investigación se observó que la producción de estructuras reproductivas en $S$. thurberi es asincrónica, ya que es común encontrar en una misma planta botones, flores y frutos al mismo tiempo. Sin embargo, observamos mayor sincronía en la población de Las Cruces, posiblemente asociada con el aumento de las temperaturas máximas de primavera; esto se corresponde con menor duración del periodo reproductivo, y corrobora lo que ya se ha dicho de las poblaciones del Desierto Sonorense. En este aspecto, la población de Las Cruces es más semejante a la población de Buenavista que a la del Plan de Guadalupe, como corresponde a su posición intermedia en el gradiente climático.

El comportamiento asincrónico de la fenología reproductiva se ha interpretado como un mecanismo para asegurar que sólo una parte de las estructuras reproductivas sean afectadas en caso de presentarse condiciones adversas de factores bióticos (plagas) o físicos (lluvias o sequías), mientras la otra parte llega exitosamente al final del ciclo (Pimienta-Barrios \& Nobel 1995, León \& Domínguez 1991); es decir, es una estrategia que permite afrontar a largo plazo la variabilidad ambiental en los hábitat que ocupa la especie. La participación del régimen de lluvia en la fenología reproductiva de $S$. thurberi podría ser indirecta, al modificar las temperaturas máximas promedio del verano; éstas parecen abonar en la duración del periodo reproductivo, mientras que los efectos directos son poco probables por su adaptación a la sequía (Bustamante \& Búrquez, 2008).

Las condiciones climáticas y las interacciones son parte de los factores que moldean el comportamiento fenológico de las poblaciones, sin embargo existen restricciones genéticas y filogenéticas por las cuales ciertos patrones son independientes de los factores ecológicos. Debido a tales restricciones, las especies de una misma familia botánica en floras ecológicamente distantes pueden compartir un alto grado de semejanza fenológica (Davies et al. 2013).

En conclusión, se registró una tendencia decreciente de la costa hacia el piedemonte en la duración y sincronía de la reproducción de las poblaciones de Stenocereus thurberi. La producción de estructuras reproductivas en la población costera es 2,5 a 4,2 veces mayor que en la población del valle y la del piedemonte, lo cual define un gradiente de estrés paralelo al gradiente de precipitación. Las poblaciones de pitaya localizadas en bosque caducifolio revisten un interés particular para investigar cómo las interacciones biológicas de la especie pueden conjugarse con las limitaciones físicas del ambiente para reproducirse y extenderse en hábitats marginales.

\section{AGRADECIMIENTOS}

El primer autor agradece al Consejo Nacional de Ciencia y Tecnología (convenio 302391) el apoyo para realizar estudios de Doctorado en el Colegio de Ciencias Agropecuarias de la Universidad Autónoma de Sinaloa. Se agradece también la gentileza de los estudiantes de la Lic. en Biología Vianey Acosta Peraza, Edith Angulo Carrillo, Omar Ramírez Ríos y José Roberto Rodríguez Martínez, por su valioso apoyo en el trabajo de campo, y las valiosas observaciones de dos revisores anónimos que contribuyeron a mejorar la calidad del artículo.

\section{REFERENCIAS}

Arriaga, L., Y. Maya, S. Diaz \& J. Cancino. 1993. Association between cacti and nurse perennials in a heterogeneous tropical dry forest in Northwestern Mexico. Journal of Vegetation Science 4: 349-356.

Blanca Mena, M. 2004. Alternativas de análisis estadístico en los diseños de medidas repetidas. Psicothema 16: 509-518.

Bustamante, O.E. 2003. Variación espacial y temporal en la reproducción y estructura poblacional de Stenocereus thurberi, una cactácea columnar del matorral costero del sur de Sonora. Tesis de maestría. Universidad Nacional Autónoma de México. México D. F. 95 pp.

Bustamante, E. \& A. Búrquez. 2008. Effects of plant size and weather on the flowering phenology of the organ pipe cactus (Stenocereus thurberi). Annals of Botany 102: 1019-1030. 
Fenología y estructura de tamaños de Stenocereus thurberi: SAlomon-MontiJo, B. ET AL.

Bustamante E., A. Casas \& A. Búrquez. 2010. Geographic variation in reproductive success of Stenocereus thurberi (Cactaceae): effects of pollination timing and pollinator guild. American Journal of Botany 97(12): 2020-2030.

Céspedes-Flores, S.E. \& E. Moreno-SÁnchez. 2010 Estimación del valor de la pérdida de recurso forestal y su relación con la reforestación en las entidades federativas de México. Investigación Ambiental 2(2): 5-13.

Cody, M.L. 1986. Distribution and morphology of columnar cacti in tropical deciduous woodland, Jalisco, México. Vegetatio 66: $137-145$

CoRnEJo, D.O. \& B.B. Simpson. 1997. Analysis of form and function in North American columnar cacti (Tribe Pachycereeae). American Journal of Botany 84(11): 1482-1501.

Cota-SÁnchez, J.H., Á. Reyes-Olivas \& B. SÁnchez-Soto. 2007. Vivipary in coastal cacti: a potential reproductive strategy in halophytic environments. American Journal of Botany 94: $1577-1581$.

DamgaArd, C. \& J. Weiner. 2000. Describing inequality in plant size or fecundity. Ecology 81(4): 1139-1142.

Davies, T.J., E.M. Wolkovich, N.J.B. Kraft, N. Salamin, J.M. Allen, T.R. Ault, J.L. Betancourt, K. Bolmgren, E.E. Cleland, B.I. Cook, T.M. Crimmins, S.J. Mazer, G.J. McCabe, S. Pau, J. Regetz, M.D. Schwartz \& S.E. Travers. 2013. Phylogenetic conservatism in plant phenology (S. Bonser, ed.). Journal of Ecology 101(6): $1520-1530$.

Díaz-Hernández, H., M.C. Navarro-Carbajal \& C.A. RodríguezMendozA. 2008. Aspectos de la morfometría y fenología reproductiva de Echinocactus platyacanthus en la Barranca Huexotitlanapa en Tecali de Herrera, Puebla. Cactáceas y Suculentas Mexicanas 53(4): 100-107.

Esparza-Olguin, L., T. Valverde \& E. Vilchis-Anaya. 2002. Demographic analysis of a rare columnar (Neobuxbaumia macrocephala) in the Tehuacan Valley, México. Biological Conservation 103: 349-359.

Figueiredo-Goulart, M. 2005. Phenological variation within and among populations of Plathymenia reticulata in Brazilian Cerrado, the Atlantic Forest and transitional sites. Annals of Botany 96(3): 445-455.

Fleming, T.H. 2000. Pollination of cacti in the Sonoran Desert. American Scientist 88: 432-439.

Fleming, T.H. 2006. Reproductive consequence of early flowering in organ pipe cactus, Stenocereus thurberi. International Journal of Sciences 167: 473-481.

Fleming, T.H., C.T. Sahley, J.N. Holland, J.D. Nason \& J.L. HAMricK. 2001. Sonoran Desert columnar cacti and the evolution of generalized pollination systems. Ecological Monographs 71: 511-530.

Fleming, T.H., M.D. Tuttle \& M.A. Horner. 1996. Pollination biology and the relative importance of nocturnal and diurnal pollinators in three species of Sonoran Desert columnar cacti. Southwestern Naturalist 41: 257-269.

Forrest, J. \& A.J. Miller-Rushing. 2010. Toward a synthetic understanding of the role of phenology in ecology and evolution. Philosophical Transactions of the Royal Society B: Biological Sciences 365(1555): 3101-3112.

Fournier, L.A. 1974. Un método cuantitativo para la medición de características fenológicas en árboles. Turrialba 24(4): 23-24.
Godinez-Alvarez. H., A. Valiente-Banuet \& L. ValienteBANUET. 1999. Biotic interactions and the population dynamics of the long lived cactus Neobuxbaumia tetetzo in the Tehuacan Valley, México. Canadian Journal of Botany 77: 203-208.

Gotelli, N.J. 2008. A Primer of Ecology. Sinauer Associates Inc. Massachusetts. 291 pp.

Gudiño, W. \& E. De la Barrera. 2014. Fenología reproductiva $\mathrm{y}$ tolerancia a temperaturas altas en Stenocereus queretaroensis. Polibotanica 37: 63-78.

Instituto Nacional de Estadística, Geografía E Informática (INEGI). 1988. Atlas nacional del medio físico. $224 \mathrm{pp}$.

KAwECKI, T.J. 2008. Adaptation to marginal habitats. Annual Review of Ecology, Evolution, and Systematics 39(1): 321-342.

León, J.L. \& R. Domínguez. 1991. Evaluación de la reproducción por semilla de la pitaya agria (Stenocereus gummosus) en Baja California Sur, México. Acta Botánica Mexicana 14: 75-87.

Leppig, G. \& J.W. White. 2006. Conservation of peripheral plant populations in California. Madroño 53(3): 264-274.

Medel-Narvaez, A., J.L. León de la Luz, F. Freaner-Martinez \& F. Molina-Freaner. 2006. Patterns of abundance and population structure of Pachycereus pringlei (Cactaceae), a columnar cactus of the Sonoran Desert. Plant Ecology 187(1): 1-14.

Mueller-Dombois, D. \& H. Ellenberg. 1974. Aims and methods of vegetation ecology. John Wiley and Sons. New York. $547 \mathrm{pp}$.

NoBel, P.S. 2002. Cacti: biology and uses. University of California Press, Berkeley. 290 pp.

PARKer, K.C. 1987. Seedcrop Characteristics and minimum reproductive size of organ pipe cactus (Stenocereus thurberi) in southern Arizona. Madroño 34(4): 294-303.

PARKer, K.C. 1988. Growth rates of Stenocereus thurberi and Lophocereus schottii in southern Arizona, Botanical Gazette 149: 335-346.

PARKER, K.C. 1989. Height structure and reproductive characteristics of senita, Lophocereus schottii (Cactaceae) in Southern Arizona. The Southwestern Naturalist 34: 392401.

PARKER, K.C. 1993. Climatic effects on regeneration trends for two columnar cacti in the Northern Sonoran Desert. Annals of the Association of American Geographers 83: 452-474.

Pavón, N.P \& O. Briones. 2001. Phenological patterns of nine perennial plants in an intertropical semi-arid Mexican scrub. Journal of Arid Environments 49: 265-277.

Pérez-González, S.B., Á Reyes-Olivas, E. García-Moya, A. Romero-Manzanares, J.R. García-Nava, G.A. LugoGarcía \& B. SÁnchez-Soto. 2015. Almacenamiento de semillas y germinación de Stenocereus thurberi, una cactácea con viviparidad facultativa. Botanical Sciences 93(2): 1-10.

PetiT, S. 2001. The reproductive phenology of three sympatric species of columnar cacti in Curacao. Jornal of Arid Environments 49: 521-531.

Pimienta-Barrios, E. \& P.S. Nobel. 1995. Reproductive characteristics of pitayo (Stenocereus queretaroensis) and their relationships with soluble sugars and irrigation. Journal of American Society of Horticultural Science 120: 
1082-1086.

Reyes-Olivas, A., E. García-Moya \& L. López-Mata. 2002. Cactishrub interactions in the coastal desert of northern Sinaloa, Mexico, Journal of Arid Environments 52: 483-497.

Rodríguez-Oseguera, A.G., A. Casas, Y. Herrerías-Diego \& E. Pérez-Negrón. 2012. Effect of habitat disturbance on pollination biology of the columnar cactus Stenocereus quevedonis at landscape-level in central Mexico, Plant Biology 15(3): 573-582.

Rojas-Sandoval, J. \& E. Meléndez-Ackerman. 2013. Population dynamics of a threatened cactus species: general assessment and effects of matrix dimensionality. Population Ecology 55: 479-491.

RondOn, J. 1994. Sinopsis de las principales metodologías aplicadas a los estudios fenológicos de los árboles tropicales. Revista Forestal Latinoamericana 14: 5-32.

Ruiz, A., M. Santos \& J. Cavelier. 2000. Estudio fenológico de Cactáceas en el enclave seco de la Tatacoa, Colombia. Biotropica 32(3): 397-407.

Rzedowski, J. 1978. Vegetación de México. Limusa. México, D.F. $432 \mathrm{pp}$.

SAHLEY, C.T. 2001. Vertebrate pollination, fruit production, and pollen dispersal of Stenocereus thurberi (Cactaceae). The Southwestern Naturalist 46(3): 261-271.

Sánchez Soto, B.H., E. García-Moya, T. Terrazas \& A. ReyesOliva. 2005. Efecto de la hidratación discontinua sobre la germinación de tres Cactáceas del desierto costero de Topolobampo, Ahome, Sinaloa. Cactáceas y Suculentas Mexicanas 50: 4-14.
Sánchez-Soto, B., A. Reyes-Olivas, E. García-Moya \& T. TERrazas. 2010. Germinación de tres cactáceas que habitan la región costera del Noroeste de México. Interciencia 35: 299-305.

SAS Institute. 2011. SAS/STAT 9.3 User's Guide. Cary, NC, USA. $8621 \mathrm{pp}$.

Siegel, S. 1985. Estadística no paramétrica. México. Trillas. 344 pp.

Soule, M. 1973. The epistasis cycle: a theory of marginal populations. Annual Review of Ecology and Systematics 4: $165-187$.

Steenbergh, W.F. \& C.H. Lowe. 1977. Ecology of the saguaro II: reproduction, germination, establishment, growth and survival of the young plant. National Park Service Scientific Monograph Series No. 8. Government Printing Office. Washington, D.C. 242 pp.

Tamhane, A.C. 2009. Statistical analysis of designed experiments, theory and applications. John Wiley \& Sons. Hoboken, New Jersey. 679 pp.

Turner, R.M., J.E. Bowers \& T.L. Burgess. 1995. Sonoran Desert plants: an ecological atlas. Tucson, AZ: University of Arizona Press. 501 pp.

WAnG, C., Y. TANG \& J. Chen. 2016. Plant phenological synchrony increases under rapid within-spring warming. Scientific Reports 6: 25460.

Yetman, D. 2006. Organ pipe cactus. University of Arizona Press. Tucson. 64 pp.

Yetman, D. \& T.R. Van Devender. 2002. Mayo ethnobotany: land, history, and traditional knowledge in northwest Mexico. University of California Press. California. 359 pp.

Recibido: 05.10 .15

Aceptado: 29.08.16 